\title{
AREUS - Innovative Hardware and Software for Sustainable Industrial Robotics
}

\author{
M. Pellicciari, A. Avotins, K. Bengtsson, G. Berselli, N. Bey, B. Lennartson, D. Meike
}

\begin{abstract}
Industrial Robotics (IR) may be envisaged as the key technology to keep the manufacturing industry at the leading edge. Unfortunately, at the current state-of-the-art, IR is intrinsically energy intensive, thus compromising factories sustainability in terms of ecological footprint and economic costs. Within this scenario, this paper presents a new framework called AREUS, focusing on eco-design, eco-programming and Life Cycle Assessment (LCA) of robotized factories. The objective is to overcome current IR energetic limitations by providing a set of integrated technologies and engineering platforms. In particular, novel energy-saving hardware is firstly introduced, which aim at exchanging/storing/recovering energy at factory level. In parallel, innovative engineering methods and software tools for energy-focused simulation are developed, as well as energy-optimal scheduling of multi-robot stations. At last, LCA methods are briefly described, which are capable to assess both environmental and economic costs, linked to the flows of Material, Energy and Waste (MEW). A selected list of industrially-driven demonstration case studies is finally presented, along with future directions of improvement.

Index Terms-Energy-Efficient Industrial Robotics, DC-grid, Computer-Aided-Robotics, Optimal Sequences, LCA.
\end{abstract}

\section{INTRODUCTION}

The fierce competition within modern globalized markets requires high-performance, reconfigurable, adaptive and evolving factories based on robotic technologies. In parallel, it is necessary to reduce factories ecological footprint by achieving a more efficient use of material and energy resources. Henceforth, it is clear that Factories of the Future will have to be smart and green [1]-[3]. On one side, the extensive use of Industrial Robotics (IR) is finally leveraging smart manufacturing. Nonetheless, on the other side, IR is intrinsically energy intensive, and its massive adoption compromises factories sustainability, not only in terms of ecological footprint but also in terms of economic costs, associated to the increasing energy prices. The main challenges to be solved in order to enable a really sustainable robotic manufacturing are related to the lack of:

- Effective solutions for reducing the energy consumption, specifically optimized for multi-robot lines and cells (i.e. maximum energy efficiency);

- Methods and computer aided tools for the eco-efficient design of sustainable IR plants;

M. Pellicciari is with the University of Modena and Reggio Emilia, Italy, e-mail: marcello.pellicciari@unimore.it; A. Avotins is with Riga Technical University, Latvia, e-mail: Ansis.Avotins@rtu.lv; K. Bengtsson and B. Lennartson are with Chalmers University of Technology, Sweden, e-mail: kristofer@sekvensa.se; bengt.lennartson@chalmers.se; G. Berselli is with University of Genoa, Italy, email: giovanni.berselli@unige.it; N. Bey is with The Technical University of Denmark, Denmark, email: niki@dtu.dk; D. Meike is with Daimler AG, Germany, e-mail: davis.meike@daimler.com.
- Automated tools for the energy optimization of IR manufacturing processes (eco-efficient process scheduling);

- Life Cycle Assessment (LCA) methods for assessing both environmental and economic costs, linked with the flows of Material, Energy and Waste (MEW).

The AREUS project [4] (Automation and Robotics for EUropean Sustainable manufacturing) aims at solving all these challenges by providing an innovative set of integrated technologies and engineering platforms, intrinsically interdisciplinary, modular and configurable. In this paper, the AREUS approach will be described, with particular focus on four main innovations:

1) Energy consumption reduction technologies, based on a novel electrical power supply system to exchange, harvest, store and recover energy at factory level, improving the use of renewable energy sources;

2) An IR integrated design and simulation environment, specifically conceived for the eco-design of IR plants and focused on the simulation of the energy flow;

3) An IR processes optimization environment, specifically conceived for the energy-optimal production scheduling and the subsequent automated computation of robot code to be fed into the Programmable Logic Controller (PLC);

4) LCA methods to assess/optimize both environmental and economic costs, linked with the MEW flows of co-evolving products and processes realized with robotic production systems.

The realization of such building blocks, whose schematic is depicted in Fig. 1, is currently enabling the modular and scalable/progressive development of optimized sustainable IR applications (or the re-adaptation of existing production facilities). Moreover, a special focus herein is placed on reducing the IR ecological footprints and the total life cycle costs, as compared to conventional factories of similar productivity rates. The paper is simply organized as follows: Sec. II to Sec. V respectively describe the four aforementioned innovations; Sec. VI provides an overlook of the demonstration activities; Sec. VII provides general observations and final remarks.

\section{IR ENERGY CONSUMPTION REDUCTION TECHNOLOGIES}

Concerning the first AREUS innovation and regarding automotive manufacturing as one of most automated industries with a high degree of IR applications, several aspects related to energy efficiency have been previously examined in collaboration with the major automotive manufacturer 

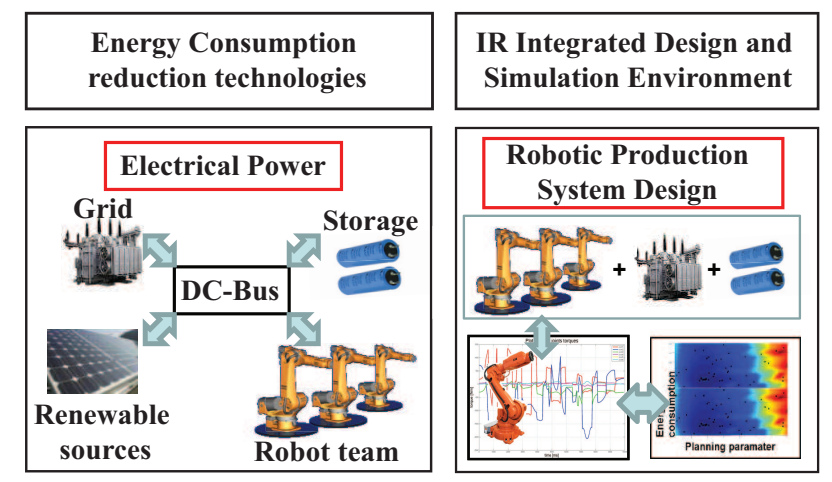
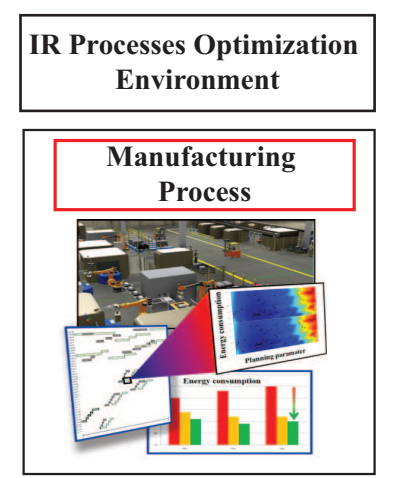

LCA Methods to Assess/Optimize Environmental/Economic Costs

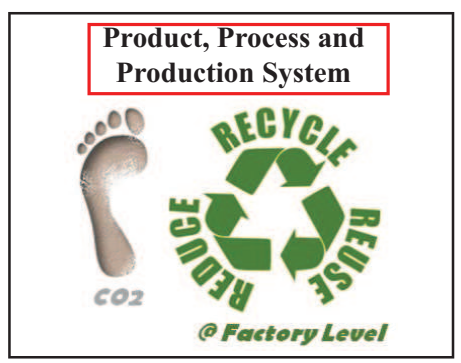

Fig. 1. Schematic of the four AREUS innovations.

Daimler AG, resulting in a doctoral thesis [5] and in experimental field testing within a real assembly plant located in Sindelfingen, Germany [6]. In addition, the vision of future manufacturing represented in the German national initiative Industrie 4.0 [7], which promotes an individualised, adaptive and fast production based on digital communication technologies, would present new functionalities also from an energy utilisation perspective.

Within this context, the AREUS project is a logical continuation of previous research in a much larger scale and, at the same time, it is dealing with new technical challenges in industrial environments. In particular, IR Energy Consumption Reduction Technologies development is based on a DC-grid electrical power supply system and a new DCRobot architecture. As explained in the following, envisaged benefits of these innovative AREUS concepts are:

- Simplified and more efficient integration of renewable power sources into factory power grid;

- Minimized loss of recuperative energy of electric drives at factory level;

- Reduction of power losses due to minimized power conversion stages and more effective energy transfer;

- Copper savings due to peak power reduction;

- Installation cost and material reduction by significantly less hardware;

- Practical enabler for realization of smart grid concepts.

\section{A. DC-Grid Electrical Power Supply System}

Nowadays, advances in power electronics enable to realize DC-grid also at higher voltages, thus promising additional power savings over conventional AC power grids due to fewer conversion stages needed and higher converter efficiency. On a factory level, which is the scale and scope of the AREUS project, similar power losses are present due to conventional AC-distribution. Furthermore, most of renewable energy sources (wind, photovoltaic, fuel cell) as well as power consumers are DC-based or have an integrated DC-link in the power converters. In addition, also energy buffer and storage devices mostly has DC-based elements, thus a more efficient choice is to use DC-DC power interface modules to connect to DC-Grid and also enable bidirectional power flow.

As a conceptual example, Fig. 2 depicts a conventional AC power system connected to a couple of AC loads (e.g. two AC electric motors). The supply of the first load requires three conversion stages (i.e. numbers 1-3 in the picture). Similarly, energy exchange among the two loads requires four conversions (i.e. numbers 4-7). On the other hand, Fig. 3 depicts the novel DC-based architecture. In such case, the supply of the first load requires a single conversion (i.e. number 1), whereas energy exchange among the two loads requires two conversions (i.e. numbers 2-3). As underlined in the same Fig. 3, the AREUS project DC-Grid voltage is selected to be $600 \mathrm{~V}$, to enable eased interconnection to existing AC grid, and to use cost-effective semiconductor materials for power interface module development.

Another issue is that electrical drives used in production may have short-time peak power requirement, up to 10 times

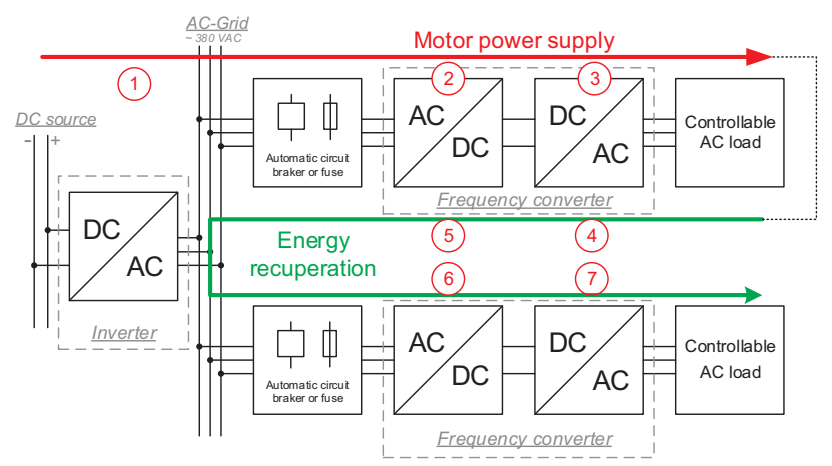

Fig. 2. State of the art AC power system (1-7 = AC/DC conversion stages).

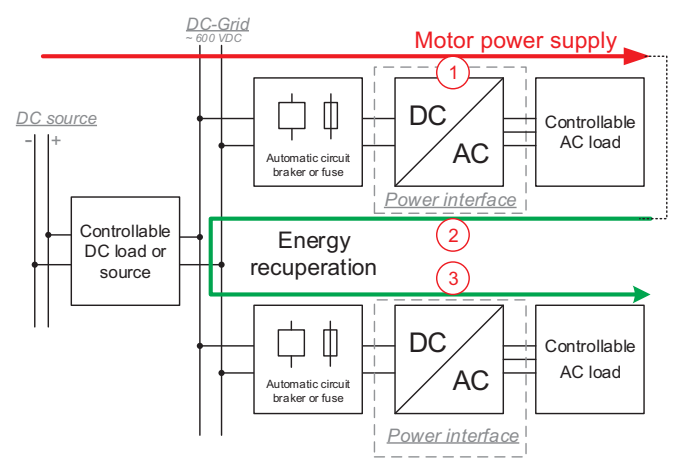

Fig. 3. DC-based power architecture (1-3 = AC/DC conversion stages). 
higher than the actual base load, which leads to infrastructure over-dimensioning and waste copper use (keeping in mind that peak energy is typically also very expensive). Modelling results shows that using AREUS DC-Grid approach with fast high power energy storage systems, combined with appropriate DC-grid interface converters, can save up to $30 \%$ of peak power requirement locally in the industrial robot manufacturing cell.

\section{B. DC-Robot Architecture}

The conventional industrial robot architecture is based on permanent magnet synchronous servo motors that are $\mathrm{AC}$ driven, and typically have quick starts, stops and rapid direction changes in the time frames often less than a second. In the latter case, during manufacturing process, braking energy is typically lost in braking resistors as heat. Similarly as for an electric car, also here recuperative energy can be re-used. Nonetheless, in this case, an AC-robot system (as described in [8]) must be used, where energy savings between 2 robots are 5-20\% with return-of-investment of 8 years.

Within the AREUS project approach, the industrial robot can be treated as either a load or even as an energy source. In the latter case, a new robot prototype, which can be powered directly from 600V DC voltage grid, has been developed, and preliminary testing results will be provided in next scientific papers and demonstration facilities of the AREUS project. As a mechanical basis, a KUKA high payload robot (Quantec 210R2700 Prime) is used, but new power electronics converter interfaces are developed, allowing DCgrid connection and bidirectional energy flow, enabling new control algorithms to be applied, that should increase energy efficiency for a given production rate.

\section{IR INTEGRATED ECO-DESIGN AND SimUlation ENVIRONMENT}

At the current state-of-the-art, IR plant design mainly focuses on product quality and production rate, robot tasks and idle configurations being developed under technological constraints only. In addition, the choice of the robot type and its positioning within the cell is based on optimal reachability and dexterity. In this context, although recent researches have practically proved that the IR power consumption can be partially reduced if energy optimality is introduced as a design goal [9], [10], most of the available ComputerAided-Robotics (CAR) and Digital Manufacturing tools do not include energy computation/optimization as a part of the plant design practice. Within this scenario, as the second AREUS innovation, a novel simulation environment has been developed, which provides a direct computation of the IR energy consumption to be made readily available to the designer. Envisaged benefits of these innovative AREUS concepts are:

- Capability to assess energy consumption of novel IR plants already during the initial design stages;

- Eco-efficient re-design of existing plants, which are far from their life cycle end.

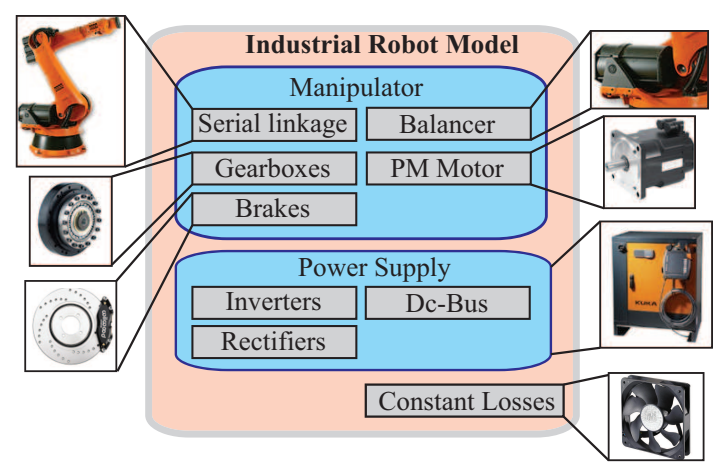

Fig. 4. Schematic of the Industrial Robot Model: Sub-system components.

\section{A. Development of an Energy-Aware CAR tool}

The new AREUS CAR tool is based on a white-box and modular model of the IR energy flow, firstly developed under a Matlab platform and then integrated into an external CAR product. For what concerns the IR mathematical model, as depicted in Fig. 4, the dynamic behaviour of all energysignificant sub-components is included, namely manipulator rigid-body dynamics, spring/pneumatic balancer, gearmotors (including normally-closed brakes), inverters, DC-Bus components, rectifier and load-independent power losses (due to cabinet's PC, cooling, and IR control panel). A detailed model derivation can be found in [11], whereas a detailed description of the parameter identification methods is presented in [12].

For what concerns commercial CAR tools, Delmia Robotics V5 has been chosen as the preferred external platform, due to its wide spread use in the automotive industry. In particular, starting from a Matlab code, the Matlab Compiler (R) has been used for the generation of a .NET Dynamic Link Library (DLL), and subsequently used by a Visual Basic software purposely developed for the interfacing with the CAR tool (Delmia V5). Then, the energy consumption of the various sub-system components is then readily computed

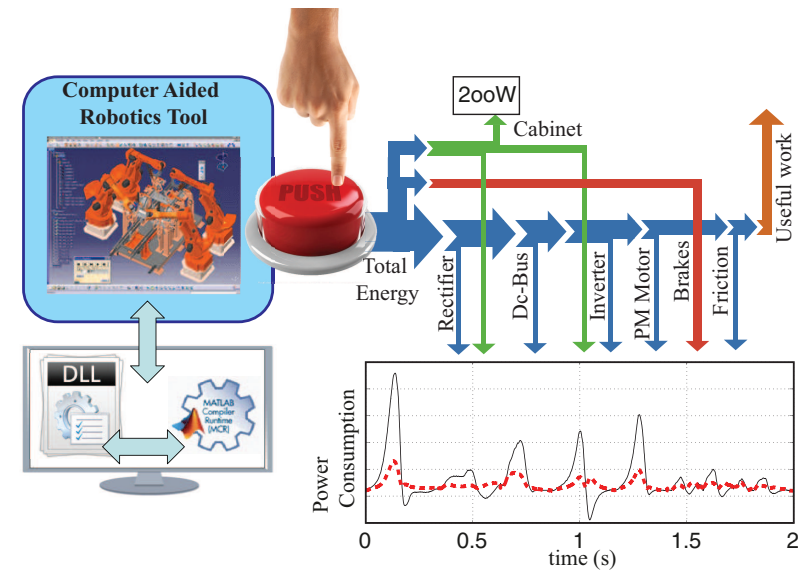

Fig. 5. Energy-aware Computer-Aided Robotics. A DLL library is interfaced with an external software (e.g. Delmia Robotics). The user can easily visualize the power losses in the various system components (i.e. rectifier/inverter/Dc-bus, Permanent Magnet (PM) electric motors, safety brakes, mechanical friction). 
once the robot electromechanical parameters are set within the DLL.

As conceptually depicted in Fig. 5, this augmented, CADbased and user-friendly, simulation environment allows to automatically visualize and highlight multi-robot energy signatures (i.e. energy consumption as a function of the main system design parameters such as cycle time, payload, DCBus capacitance, and other critical electromechanical components [8]). By noticing that industrial robots are typically programmed for applications of cycle times from several seconds to a minute or more, that are repeated millions of times during the whole production life cycle, it becomes clear that even a very little energy optimization of a particular robot application may achieve significant savings. In its future implementation, this energy-aware CAR tool will also provide information about energy-optimal robot base positioning and robot selection.

\section{IR PROCESSES ECO-OPTIMIZATION}

Robot movements are often optimized focusing on time performance. More recently, energy consumption has also been an issue. The third AREUS innovation therefore focuses on

- Energy-efficient performance via optimal robot trajectories;

- Efficient computation of energy optimal sequences of operations (scheduling) for multi-robot systems, still keeping desired cycle times.
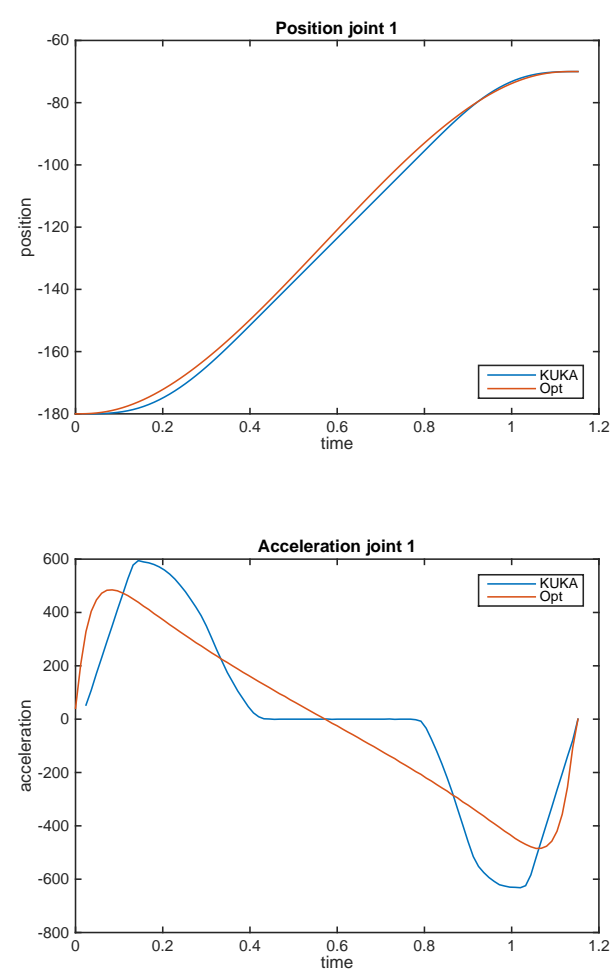

\section{A. Energy-Optimal Trajectories}

A simple but novel concept for generation of energyoptimal robot trajectories has recently been developed and presented in [13]. As a starting point, manually defined robot paths are generated by the normal robot programming language. The resulting robot trajectories, sampled (about $100 \mathrm{~Hz}$ ) and recorded either from physical robots or a CAR simulation tool, are delivered to an optimization algorithm. The original equidistant sampling times are then adjusted by the optimizer to simply minimize a weighted sum of squared accelerations on each robot joint. At the same time the original robot paths are preserved, while velocities and accelerations are changed due to the modified sampling times. The resulting optimization problem is nonlinear and non-convex, but solved quite efficiently due to the good initial solution given by the manually defined robot paths. The optimized trajectories are converted to trajectory control code that is possible to directly execute by KUKA-robots.

A typical result is shown in Fig. 6, where the first joint for a real KUKA robot KRC30 is moving 110 degrees with $100 \%$ speed. The difference between the original trajectory generated by KUKA's standard robot commands (blue curves) and the optimal solution (red curves) is shown in this figure. After the initial acceleration and before the final retardation, the optimal solution generates a constant jerk (derivative of the acceleration), see the red curve in the acceleration diagram in Fig. 6, instead of the original constant speed, see the blue curve in the velocity diagram. This difference saves about $20 \%$ of energy for this simple
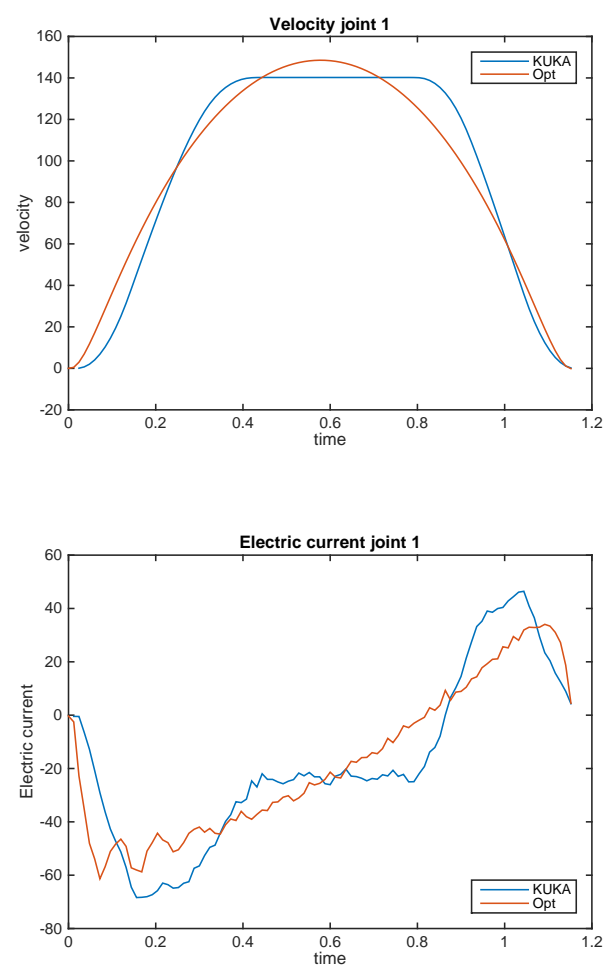

Fig. 6. Comparison of angular positions, velocities, accelerations, and electric currents before (blue) and after optimization (red). 
scenario. Observe that the optimized trajectory has the same total execution time as the original one. The optimized trajectories have a higher jerk and sometimes also a higher maximum speed compared to the original motion, but it is within limits for what is possible with the robots. Generally the optimized motions looks and feels smother and less aggressive.

In another example a longer path has been optimized, including multiple points and both point-to-point and linear motions. The result for the optimized version is, in this case, an impressive $32 \%$ energy reduction, ones again the same total execution time for the original and the optimal trajectories.

\section{B. Energy-Optimal Sequences for Multi-Robot Systems}

The optimization strategy above can also be generalized to multi-robot systems, where robots also need to be coordinated to avoid collisions. This concept has been implemented in the new AREUS Sequence Planner (SP) [14], [15], which also includes specific algorithms for combined multi-robot scheduling and energy optimization. Previous research in energy-optimal sequencing, along with the determination of energy-efficient trajectories in the presence of robots with shared workspaces (shared zones), can be found in e.g. [9], [16], [17]. For an excellent survey of existing approaches, see [18]. Following a similar direction, including the new trajectory optimization in Section IV-A, the new algorithms implemented in the AREUS SP focuses on minimizing the acceleration, while assuring practically feasible IR sequences.

To illustrate this multi-robot concept, a real system composed of two robots has been considered, where robot R1 is moving down and R2 is moving up in a shared zone. To avoid collision, both robots cannot be in the shared zone at the same time. The order in which the shared zone should be passed is not defined in the original motion. Thus, two possible sequences are identified:

- Sequence S1: $R_{1}$ starts a downward motion, followed by $R_{2}$ moving up.

- Sequence $S 2$ : Similar to $\mathrm{S} 1$, but $R_{2}$ starts first.

The results of this experiment when optimizing the trajectories show that for both sequences, almost $30 \%$ of the energy consumption of the two robots was saved by the optimized and coordinated motions. The results also show that the second sequence (S2), where $R_{2}$ moves through the shared zone first, consumes less energy. When comparing an original motion, where $R_{1}$ moves first, with the solution that the optimizer generates, where it is better to start moving $R_{2}$, the final optimized trajectories and coordination saves $45 \%$ of the energy.

In this example only two sequences were identified. Generally, many sequences may be included in an optimization loop, where each sequence is tested separately. By including efficient search techniques, involving for instance constraint programming, this exhaustive search can be reduced significantly by integrated optimization methods [19], [20].
To summarize: energy can be saved by 1) optimizing the acceleration in the trajectories, 2) allowing robots to move slower instead of waiting for shared zone access, and 3) changing the sequences of operations, see further details in [13].

\section{Methods For IR Life Cycle Assessment}

When in use in industrial robotic factories, the abovedescribed innovative AREUS technologies, as well as technologies in general, are always linked with MEW flows. These MEW flows define the sustainability performance profile of a selected configuration, and in AREUS, this sustainability performance is quantified in terms of environmental and economic impact. In combination with a tailor-made method for the economic appraisal, AREUS employs LCA, the internationally standardized state-of-the-art methodological framework to assess environmental sustainability [21].

In order to effectively support planning and re-/design of robotic factories, a fourth AREUS innovation consists of an integrated method to assess and optimize both environmental and economic consequences of design/technology choices. By means of this method, which will be integrated in a professional software environment, the overall sustainability improvement of a given option can be quantified already in the planning stage. This approach thus addresses an issue which in earlier research has been identified to be a major barrier for implementing environmental strategies in manufacturing companies, e.g. [22]. Main output of the assessment methodology will be one single figure for the environmental performance, represented as a so-called Carbon Footprint (i.e. the sum of contributions to Global Warming) and one single $€$ figure for the economic performance.

Valid sustainability performance assessment is not possible without stating exactly which elements of a production system are included and which not. However, robotic manufacturing systems most typically consist of several interlinked systems and sub-systems, e.g. [23], and system delimitations are often not clearly specified. As part of the integrated assessment method, this AREUS innovation also clearly describes different types of robotic manufacturing systems and maps Key Performance Indicators for them. The three major types of systems distinguished are:

- One robot KR 210 Quantec including control unit;

- One manufacturing cell consisting of 4 robots, welding, gluing and handling technologies and other equipment;

- One production line consisting of 8 (TBC) manufacturing cells and other equipment.

The AREUS system delimitations are defined in view of the different perspectives that the various stakeholders take during the design of robotic production lines, so that stakeholders are enabled to make sustainability-oriented decisions in their individual scope of activity. The decision flow among stakeholders is shown in Fig. 7 with the assessment tool in the center. In order to provide for fair overviews, the same system delimitations are applied for the environmental assessment (LCA) as for the economic appraisal.

As a final crucial element in the AREUS sustainability 


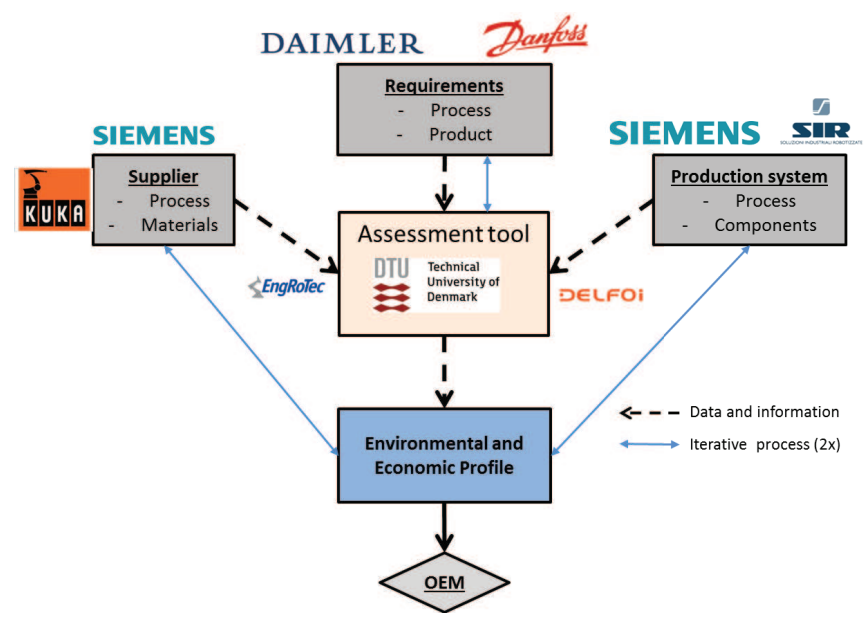

Fig. 7. Information flows among AREUS partners as example for the integration of the AREUS assessment tool in the planning process for sustainable robotic factories.

assessment approach, the entire life cycle of the manufacturing systems is considered. Together with the clear distinction of system levels, this life cycle view ensures that potential sub-optimisations or burden shifts can be avoided when making the assessment. Thus, it becomes visible whether, for instance, a possible material choice that reduces impacts in the use stage of a robot may lead to substantially increased impacts in the disposal stage or whether a certain technology choice on the production cell level may result in increased impacts at a higher level, e.g. at the overall production line level. This total system-wide and total life cycle-wide approach is adopted in AREUS to ensure best-inclass sustainability performance of the developed solutions.

\section{Vi. Demonstration Case Studies}

The AREUS methodology is experimentally validated in various real production and test environments. The interrelation is shown in Fig. 8, where software optimization is strongly related to novel hardware concepts. For instance, peak power reduction is achieved by both energy storage/exchange technology and methods for energy flow simulation, monitoring and control.

In AREUS, there are seven demonstration facilities being developed in six different countries. Four of them are physical laboratories and three are virtual or cyber-physical demonstration stations. A demonstration laboratory is a physical manufacturing unit, a prototype that is not used for operative production, however, capable to demonstrate real production processes on real equipment. Virtual laboratory is generally software, specific model or a software-tool-chain to demonstrate off-line solutions for manufacturing planning, optimization and evaluation. An overview of the AREUS demonstration facilities is given in Table I. Each demonstration facility has a specific location and a respective project partner, responsible for development and management of this facility.

Virtual Production design demonstration lab (Demo 1) takes place at the Mercedes-Benz production technology center in Sindelfingen, Germany. It demonstrates the AREUS eco-design principles via virtual engineering tools to reduce material use, construction costs and increase energy efficiency simultaneously. All the AREUS optimization approaches are planned to be implemented into production planning software tools that have a large market share so that the AREUS software modules become de facto ingredients for the production planning engineers and system integrators.

A demonstration cell with four robots and tooling (Demo 2) is a copy of a real robot cell from the existing car production. This cell has a capability to produce real car parts, however with a novel hardware design, based on DC energy system, enabling a natural energy exchange between equipment and eased energy storage. This particular demo cell is named Smart Automation Grid. AREUS aims to demonstrate novel technological concepts in maturation

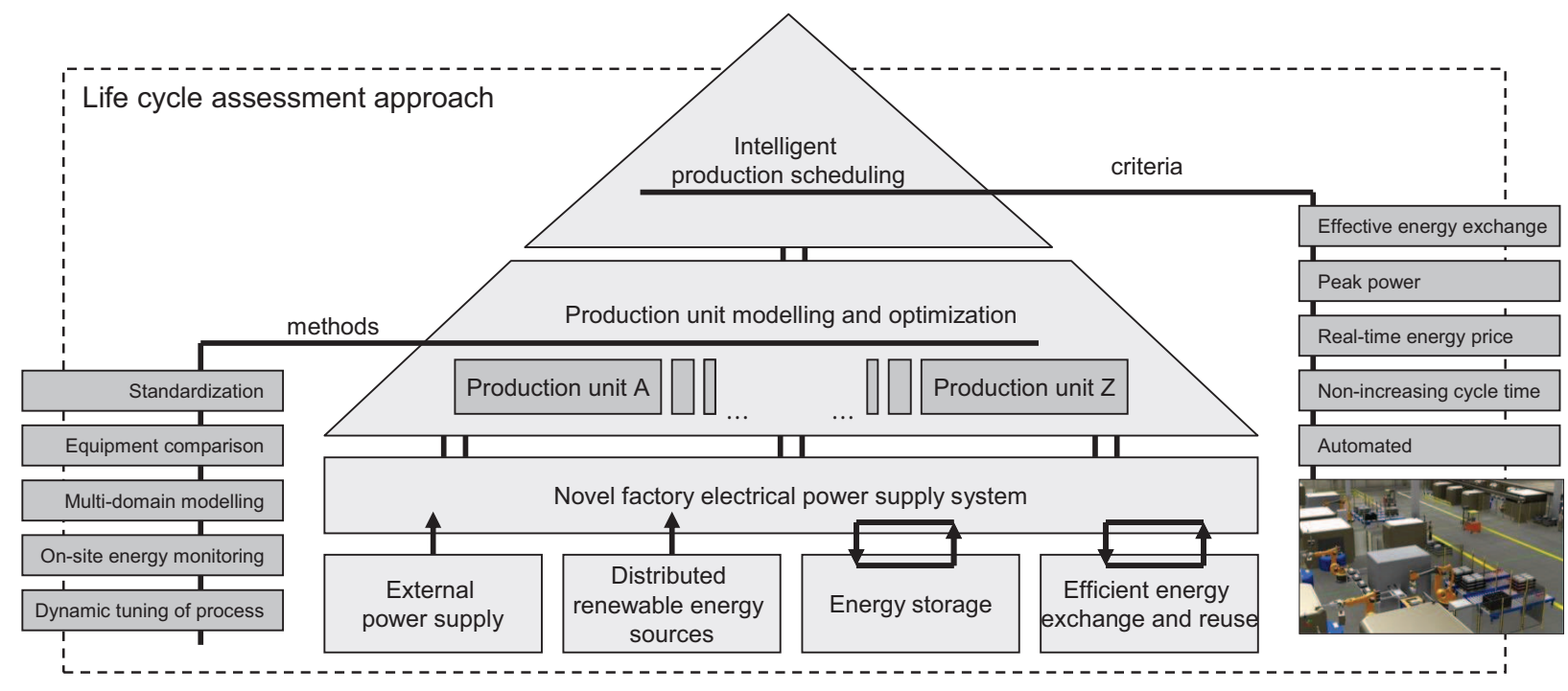

Fig. 8. Interrelation of various AREUS production optimization concepts for highly automated robotic factories. 
TABLE I

OVERVIEW OF THE AREUS DEMONSTRATION FACILITIES

\begin{tabular}{|c|l|c|}
\hline 1 & Virtual production design demonstration lab & Germany \\
\hline 2 & $\begin{array}{l}\text { Demonstration cell with 4 robots and tooling based on novel } \\
\text { power supply system }\end{array}$ & Germany \\
\hline 3 & $\begin{array}{l}\text { Demonstration cell with 5 industrial robots and conveyer system: } \\
\text { intelligent production scheduling methods and idle mode control }\end{array}$ & Sweden \\
\hline 4 & $\begin{array}{l}\text { Demonstration cell with 3 various payload robots: re-design and } \\
\text { re-adaptation of existing plants }\end{array}$ & Italy \\
\hline 5 & $\begin{array}{l}\text { Virtual robotic production system optimization demonstration test } \\
\text { bench }\end{array}$ & Finland \\
\hline 6 & $\begin{array}{l}\text { Industrial robot test bench with prototypes: energy exchange, } \\
\text { storage, integration of renewable energy sources }\end{array}$ & Latvia \\
\hline 7 & $\begin{array}{l}\text { Virtual lab for LCA/LCC determination for evaluation of the environmental } \\
\text { impact \& economic appraisal of robotic production plants }\end{array}$ & Denmark \\
& \multicolumn{2}{|c}{ Laboratory } \\
\cline { 2 - 3 } & \multicolumn{2}{|c|}{ Virtual lab } \\
\hline
\end{tabular}

level, never experimentally validated before. The cell shows the electrical energy saving potential, higher efficiency integration of renewable power resources and energy storage principles at field level.

A demonstration cell (Demo 3) in Sweden is an existing fully automated assembly station adapted for AREUS, including five industrial robots, conveyor system, and smart automated guided vehicle with a centralized PLC control. This demonstration focuses on sequence control and energy reduction by optimizing the robot movement paths.

In the demonstration cell (Demo 4) in Italy the previous mentioned virtual design tools are experimentally being tested. Principal equipment includes three ABB robots, electrical press, machining tools, screwdriver tools, vibrating feeders, and an energy storage system. The demonstration will focus on AREUS approaches concerning robot system modeling and control method optimization, applicable to existing production configurations.

A 3D virtual factory model (Demo 5) and a scenario were created to demonstrate the integration of enhanced LCA methods into factory planning software for operative use. When processing components, many machines generate waste, the amount of which depends on process parameters that can be modeled. Other important parameters identified to be integrated in the factory model are the transport of components and, in particular, the factory floor area/space that is needed to produce products. This is crucial since e.g. the energy required for heating, cooling, lighting etc. of factories (collectively referred to as overhead energy) typically has a substantial share in the environmental impact of a facility.

An experimental laboratory (Demo 6) is electrically representing a manufacturing work cell built with a purpose to experimentally validate the algorithms for power smoothing and energy consumption reduction technologies. It is a DC power supply system in which energy could be exchanged, harvested, stored and recovered at a factory level.

Primary objective of the virtual lab for LCA (Demo 7) is to assess the environmental and economic sustainability aspects of robotic production plants of any size. In the lab, different design options for robotic production units can be compared in order to find the option that performs best sustainability-wise. In this context, robotic production units are to be understood either as single robots, as production cells or as production lines containing robots.

\section{GENERAL OBSERVATIONS}

In this paper, a brief overview of the AREUS framework has been reported. As previously mentioned, AREUS main motivations arise from the need of tools and methods able to leverage the massive adoption of robotic technologies in the manufacturing industries. Several stakeholders may profit, such as:

- Robot technology providers, that aim at reducing the energy consumption of their robots, especially when working in multi-robot lines.

- Engineering software providers, that are interested in the development of novel tools, able to push forward the state-of-the-art of robot manufacturing systems design and optimization practices, currently focused only on mere production performance;

- Systems integrators/robotic plants builders, that are asking for the technologies needed to optimize the sustainability and shorten the payback period of IR plants;

- Engineering services companies, that require novel methods for the predictive planning and optimization of operating expenses and eco sustainability, in order to estimate with higher accuracy real business costs and cash flows.

- Final users, that would massively adopt robotic manufacturing systems for their superior and unique performances, but they need short payback periods including lower energy cost to afford them.

In order to assess these industrial needs, AREUS is developed with a modular approach to completely exploit its potential in different fields and with various levels of implementation (for example new factories, re-adaptation of existing facilities or different levels of investment affordable by the final user). In fact, each of the four AREUS innovations can be effectively used as standalone technologies or synergically integrated with each of the other modules to gain the best results with a leveraging effect. This strategic approach has been adopted not only to increase the future market exploitation opportunities, but also to foster a stepby-step and scalable approach to guide the market into progressive and easier-to-implement levels of sustainability improvements.

For what concerns potential advantages for future robotic plants, AREUS integrated technological platforms aim at achieving an average robotic plant energy consumption reduction up to $35 \%$ (in its full configuration), a peak power reduction of $50 \%$ and, at the same time, a reduction of $25 \%$ in terms of the total life cycle costs of factories with respect to conventional factories of similar productivity rates.

Naturally, although energy efficiency (and improved use of renewable energy), is the primary aim of the project, the best and more effective results cannot be achieved without a proper integration with the mutually interacting contributions of renewable materials and waste/pollutant management. 


\section{ACKOWLEDGMENTS}

This research was funded by the European Communityłs Seventh Framework Programme under grant agreement No. 609391 (AREUS).

\section{REFERENCES}

[1] European Commission. Energy Efficiency Plan. Brussels, 2011.

[2] ManuFuture. Strategic research agenda, report of the high level group. ManuFuture Platform, 2006.

[3] U. Heisel and M. Meitzner. Progress in reconfigurable manufacturing systems. J. for Manufacturing Science and Production, 6(1-2), 2011.

[4] European Commission. AREUS - Automation and Robotics for European Sustainable Manufacturing. www.areus-project.eu, 2013.

[5] D. Meike. Increasing Energy Efficiency of Robotized Production Systems in Automobile Manufacturing. PhD thesis, Riga Technical University, 2013.

[6] D. Meike, A. Senfelds, and L. Ribickis. Power converter for dc bus sharing to increase the energy efficiency in drive systems. Industrial Electronics Society, IECON 2013 - 39th Annual Conference of the IEEE, pages 7199-7204, 2013.

[7] Industrie 4.0 - http://www.bmbf.de/de/9072.php. 2014.

[8] D. Meike and L. Ribickis. Recuperated energy savings potential and approaches in industrial robotics. In The IEEE 15th Int. Conf. on Automation Science and Engineering, pages 299-303, 2011.

[9] O. Wigstrom, B. Lennartson, A. Vergnano, and C. Breitholtz. Highlevel scheduling of energy optimal trajectories. IEEE Transactions on Automation Science and Engineering, 10(1):57 -64, 2013.

[10] M. Pellicciari, G. Berselli, and F. Balugani. On Designing Optimal Trajectories for Servo-Actuated Mechanisms: Detailed Virtual Prototyping and Experimental Evaluation. IEEE/ASME Transactions on Mechatronics, PP(99):1-14, 2014.

[11] D. Meike, M. Pellicciari, and G. Berselli. Energy efcient use of multirobot production lines in the automotive industry: Detailed system modeling and optimization. IEEE Transactions on Automation Science and Engineering (available online), pages 1-12, 2013.

[12] E. Oliva, G. Berselli, and M. Pellicciari. A novel engineering method for the power flow assessment in servo-actuated automated machinery. 24th International Conference on Flexible Automation and Intelligent Manufacturing (FAIM), pages 1-8, 2014.
[13] S. Riazi, K. Bengtsson, O. Wigström, E. Vidarsson, and B. Lennartson Energy optimization of multi-robot systems. In Proc. IEEE Int. Conf. on Automation Science and Engineering (CASE), Aug 2015.

[14] K. Bengtsson and B. Lennartson. Flexible specification of operation behavior using multiple projections. Automation Science and Engineering, IEEE Transactions on, 11(2):504-515, April 2014.

[15] B. Lennartson, K. Bengtsson, C. Yuan, K. Andersson, M. Fabian, P. Falkman, and K. Akesson. Sequence planning for integrated product, process and automation design. Automation Science and Engineering, IEEE Transactions on, 7(4):791-802, Oct 2010.

[16] A. Vergnano, C. Thorstensson, B. Lennartson, P. Falkman, M. Pellicciari, F. Leali, and S. Biller. Modeling and optimization of energy consumption in cooperative multi-robot systems. Automation Science and Engineering, IEEE Transactions on, 9(2):423-428, April 2012.

[17] S. Bjorkenstam, D. Gleeson, R. Bohlin, J.S. Carlson, and B. Lennartson. Energy efficient and collision free motion of industrial robots using optimal control. In IEEE Int. Conf. on Automation Science and Engineering (CASE), pages 510-515, Aug 2013.

[18] Paryanto, Matthias Brossog, Martin Bornschlegl, and Jrg Franke. Reducing the energy consumption of industrial robots in manufacturing systems. The Int. J. of Advanced Man. Tech., pages 1-14, 2015.

[19] O. Wigström and B. Lennartson. An integrated CP/OR method for optimal control of modular hybrid systems. In Proc. 12th IFACIEEE International Workshop on Discrete Event Systems (WODES14), Cachan, France, May, pages 485-491, 2014.

[20] J.N. Hooker. Integrated Methods for Optimization. Springer, New York, NY, 2010.

[21] ISO (International Standards Organization). Iso 14040:2006: Environmental management, life cycle assessment, principles and framework. Brussels (Belgium): CEN, 2006.

[22] N. Bey, Hauschild M.Z., and McAloone T.C. Drivers and barriers for implementation of environmental strategies in manufacturing companies. CIRP Ann - Manuf. Technol., 62(1):43-46, 2013.

[23] J.R. Duflou, J.W. Sutherland, D. Dornfeld, C. Herrmann, J. Jeswiet, S. Kara, M. Hauschild, and K. Kellens. Towards energy and resource efficient manufacturing: A processes and systems approach. CIRP Annals Manufacturing Technology, 62(2):587-609, 2012. 